\title{
Editorial
}

\section{Influenza Outbreaks in Long-Term-Care Facilities: How Can We Do Better?}

\author{
Andrew E. Simor, MD, FRCPC, FACP
}

Despite the availability of influenza vaccines for several decades, infection caused by influenza viruses continues to cause considerable morbidity and mortality. Epidemics of influenza occur each year during the winter months in the northern hemisphere, accounting for excess mortality and increased hospitalization rates. ${ }^{1-3}$ Most severe disease occurs in the elderly, with approximately $90 \%$ of the deaths associated with influenza occurring in those older than 65 years. ${ }^{2}$ Residents of long-term-care facilities (LTCFs) are especially vulnerable because of their increased age and frailty and the presence of multiple comorbidities. Moreover, they live in a closed environment in proximity to other residents and have frequent contact with staff, volunteers, and visitors who may introduce influenza to the facility from the community. When influenza occurs in a nursing home, attack rates among residents may be as high as $25 \%$ to $60 \%$ with case-fatality rates of $10 \%$ to $20 \%{ }^{47}$ Although most disease is caused by influenza A virus, influenza type $B$ has also been associated with considerable morbidity and mortality. 810

Several guidelines and recommendations for influenza prevention and control in LTCFs for the elderly have been published in the past 10 years, including two that appeared in Infection Control and Hospital Epidemiology. ${ }^{11-13}$ These guidelines have all emphasized the importance of annual influenza vaccination of residents and staff, surveillance for respiratory tract infections, access to rapid influenza diagnostic testing, and policies and procedures for outbreak management, including the use of postexposure chemoprophylaxis with antiviral agents.

Annual vaccination of residents continues to be the main priority of preventive strategies. Resident vaccination in LTCFs has reduced influenza-associated pneumonia, hospitalization, and mortality rates. ${ }^{14-17}$ Nevertheless, influenza immunization rates remain suboptimal in many LTCFs. ${ }^{10,18,19}$ More recently, vaccinating nursing home staff, and not just residents, has been recognized as an important factor in reducing the risk of influenza and its complications among residents. ${ }^{20,21}$

Nursing homes and other LTCFs must be prepared in advance to deal with the possible occurrence of an influenza outbreak. There must be policies in place regarding influenza surveillance and diagnosis, appropriate isolation measures, and the potential use of postexposure chemoprophylaxis with antiviral agents. ${ }^{13,19}$ Unfortunately, few studies have been conducted to provide data on which to base such policy development. For example, the strength and quality of the evidence for almost all of the specific recommendations made by the Long-Term-Care Committee of the Society for Healthcare Epidemiology of America for influenza surveillance and outbreak management in LTCFs was classified as IIIB, indicating only "moderate evidence to support the recommendation" based on "evidence from opinions of respected authorities, clinical experience, descriptive studies, or reports of expert committees. ${ }^{13}$ Two articles in this issue of Infection Control and Hospital Epidemiology contribute to our knowledge of influenza management in LTCFs. The study by Drinka et al. addresses the issue of how to define the presence of an influenza outbreak in an LTCF in order to be able to effectively intervene with postexposure antiviral prophylaxis. ${ }^{22}$ Hirji et al. describe their experience with zanamivir, a neuraminidase inhibitor, for treatment and prophylaxis during concomitant outbreaks due to influenza A and influenza B in an $\mathrm{LTCF}^{23}$

Surveillance definitions for the identification of influenza-like illness in LTCF residents have been proposed. ${ }^{11,12,24}$ However, none of these definitions have been

Dr. Simor is from the Department of Microbiology and the Division of Infectious Diseases, Department of Medicine, Sunnybrook and Women's College Health Sciences Centre, University of Toronto, Toronto, Ontario, Canada.

Address reprint requests to Dr. Andrew E. Simor, Department of Microbiology, Sunnybrook and Women's College Health Sciences Centre, B1212075 Bayview Avenue, Toronto, Ontario M4N 3M5, Canada. 
validated in prospective studies, and in some investigations influenza case definitions were found to lack sensitivity or specificity. ${ }^{25-28}$ As a result, it is not always clear when an influenza outbreak should be declared. Definitions based on the number or proportion of cases on a unit during a specified period of time have been used to indicate the presence of an outbreak. ${ }^{11 \cdot 13}$

In this issue, Drinka et al. describe their 6-year experience in a large skilled nursing facility with four residential buildings in order to better understand factors that may have contributed to delayed implementation of chemoprophylaxis in the presence of an influenza outbreak. ${ }^{22}$ Active surveillance for influenza and other respiratory tract infections was conducted each year throughout influenza season. The threshold for beginning antiviral prophylaxis on a nursing unit was the detection of influenza $\mathrm{A}$ by culture and if $10 \%$ of the residents on that floor had new respiratory symptoms within a 7-day period. A delay in starting prophylaxis was determined to have occurred if there were four or more residents with positive cultures on a floor whose specimens had been collected within 5 days before the start of prophylaxis. During the 6 years of study, 14 clusters of delayed onset of prophylaxis were identified. The authors considered this result to be suboptimal. In six clusters there was probably little that could have been done to start prophylaxis earlier, as the outbreak was explosive with multiple culture-positive cases involving several nursing units within a short period of time. However, in five clusters (all occurring in other buildings of the facility following previously identified outbreaks) prophylaxis could have been started sooner if the threshold for starting antiviral medications had been modified. In three clusters, implementation of chemoprophylaxis was delayed while waiting for positive viral culture results.

Although it may be true that sporadic influenza virus infection may occur in an LTCF without causing an outbreak of respiratory illness, the propensity of this agent to cause widespread and severe infection in this patient population indicates that it would be prudent to have a low threshold for declaring the presence of an outbreak. A single laboratory-confirmed case of influenza in the presence of any temporal or geographic clustering of cases with new respiratory symptoms (with or without fever) should indicate the need for outbreak control measures, including provision of chemoprophylaxis. Moreover, if influenza has already been confirmed involving a nursing unit of an LTCF, a respiratory outbreak elsewhere in the facility should be investigated promptly with a lower threshold for starting prophylaxis, as noted by Drinka et al. ${ }^{22}$

This study also highlights the limitation of viral cultures in outbreak identification and management. Viral isolation may provide useful epidemiologic information, but this typically takes several days. In the setting of a possible outbreak, a more rapid diagnosis is required. Commercial test kits for detection of influenza viral antigens in clinical specimens (eg, nasopharyngeal swabs) by direct fluorescent antibody or enzyme immunoassay have been found to have sensitivities of $87 \%$ to $100 \%$ and specificities of greater than $90 \%$ as compared with viral isola- tion. ${ }^{29,30}$ It is possible to obtain same-day test results, and the utility of these diagnostic tests for the effective management of influenza outbreaks in LTCFs has been demonstrated. ${ }^{30}$ Provisions for the availability of such diagnostic testing in LTCFs should be made prior to the onset of influenza season each year. ${ }^{13}$

Once an influenza outbreak has been identified in an LTCF, several control measures should be implemented, including the provision of antiviral prophylaxis. ${ }^{3,11-13}$ Postexposure prophylaxis is recommended to reduce the transmission of influenza between residents. Until recently, the only medications available were amantadine and rimantadine. These agents have activity against influenza A viruses, but not against influenza $B$ viruses. Although these drugs have been used effectively in managing influenza $A$ outbreaks in LTCFs, ${ }^{3033}$ amantadine use has been associated with significant adverse reactions, especially confusion, delirium, ataxia, falls, and seizures. ${ }^{34,35}$ These side effects appear to occur less often with rimantadine. ${ }^{36}$ Amantadine- and rimantadine-resistant strains have also been recognized, and have been associated with failure of treatment and prophylaxis. ${ }^{3740}$

More recently, neuraminidase inhibitors such as zanamivir and oseltamivir have become available for the treatment and prevention of influenza caused by both type $A$ and type $B$ viruses. The two drugs differ primarily in how they are administered: zanamivir is administered by oral inhalation, whereas oseltamivir is ingested as an oral preparation. Zanamivir and oseltamivir appear to be equally effective in the treatment of influenza A and influenza B when started within 48 hours of the onset of symptoms. ${ }^{41-45}$ These drugs have shown efficacy as "seasonal" prophylaxis in community studies $^{46,47}$ and as postexposure prophylaxis in families. ${ }^{48,49}$ Although resistance to zanamivir and oseltamivir can be induced in influenza viruses, this appears to be infrequent. ${ }^{50-52}$

The study by Hirji et al. in this issue contributes to our knowledge about the use of neuraminidase inhibitors during influenza outbreaks in LTCFs. ${ }^{23}$ In this noncomparative study, zanamivir was provided for both treatment and prophylaxis, as appropriate, to elderly patients on a complex continuing care unit of an LTCF who were exposed to both influenza $A$ and influenza $B$ during an outbreak of respiratory infections in March 1999. Most of these patients had received influenza vaccine, and had also been given postexposure prophylaxis with amantadine in the preceding few weeks. Overall, $85 \%$ of the patients on the unit were able to complete the prescribed course of orally inhaled zanamivir with few adverse effects. As in previous studies, those unable to use the Diskhaler (GlaxoSmithKline, Toronto, Ontario, Canada) for zanamivir inhalation were easily identified by LTCF staff as being confused, disoriented, or totally dependent for assistance with activities of daily living. ${ }^{53,54}$ To determine the efficacy of prophylaxis with zanamivir for the prevention of influenza-like illness, the authors used historical data for an estimate of expected influenza attack rates. They attempted to correct for the protective effects of influenza vaccination and amantadine chemoprophylaxis. The efficacy of zanamivir prophylaxis was also calculated using attack rates on two other units in 
the facility experiencing influenza outbreaks in the preceding 4 weeks. The efficacy of zanamivir in preventing influenza-like illness in the at-risk patient population was estimated to be $82 \%$ based on historical data, and $91 \%$ as compared with the two contemporaneous control units in the facility. The authors acknowledge the important limitations of using historical data and nonmatched contemporaneous control populations, but these results appear to be consistent with the efficacy of postexposure prophylaxis with neuraminidase inhibitors found in other studies. ${ }^{46,48,49}$

Few other studies describing the use of neuraminidase inhibitors in nursing homes are currently available. Two noncomparative studies documented the safety and efficacy of zanamivir in preventing transmission of influenza A following identification of outbreaks in nursing homes in southern Ontario ${ }^{53,54}$ In one of these facilities, zanamivir was started after failure to control the outbreak because of the emergence of amantadine resistance in influenza virus isolates. ${ }^{53}$ Bowles et al. described outbreak management with oseltamivir during 11 influenza A outbreaks in 10 LTCFs in Ontario in $1999-2000 .^{55}$ In some LTCFs, oseltamivir was started after amantadine failed to control the outbreak, whereas in other facilities the drug was used as primary prophylaxis. Use of oseltamivir was associated with termination of the outbreak in all of the evaluable outbreaks. Peters et al. investigated the use of oseltamivir for seasonal prophylaxis in 31 LTCFs in the United States and Europe during the 1998-1999 influenza season. ${ }^{56}$ Once influenza activity was identified in the surrounding community, residents in the LTCFs were randomized to receive a 6 -week course of either oral oseltamivir or placebo. Oseltamivir use resulted in a $92 \%$ reduction in the incidence of laboratory-confirmed clinical influenza and a decrease in certain secondary complications. Oseltamivir given daily for 6 weeks was generally well tolerated, with few adverse reactions or drug interactions.

There is only one published randomized, controlled trial involving a neuraminidase inhibitor for the management of an influenza outbreak in an LTCF: zanamivir was compared with rimantadine for postexposure prophylaxis during the influenza season of 1996-1997 in an LTCF with an influenza A outbreak. ${ }^{57}$ In this study, zanamivir was also compared with "standard care" without drug prophylaxis during a subsequent influenza B outbreak in the facility. Zanamivir was well tolerated in the small number of residents who agreed to participate in the study, and it appeared to be effective in controlling transmission of both influenza $\mathrm{A}$ and influenza $\mathrm{B}$. However, the study did not have adequate power to determine whether one drug was better than the other. Since then, this trial has been extended to involve nine LTCFs during three influenza seasons. Preliminary results indicated that zanamivir was superior to rimantadine in preventing laboratory-confirmed symptomatic influenza A infection (relative risk, $0.39 ; P=.038)^{58}$; too few subjects were randomized during influenza $B$ outbreaks for analysis. Whereas rimantadine resistance in viral isolates was detected, there was no emergence of zanamivir resistance.
The potentially devastating impact of influenza outbreaks in LTCFs for the elderly has been recognized for many decades. Several guidelines for influenza prevention and control have been published. However, few of these recommendations are evidence based, and it is clear that there is still much room for improvement in the recognition and management of this infection. There is considerable evidence to support the effectiveness of influenza vaccination, and influenza prevention in LTCFs begins with the immunization of residents and staff. However, better strategies to improve vaccine uptake, particularly among healthcare providers, are required. The staff of LTCFs are challenged to make an accurate clinical diagnosis of influenza in elderly nursing home residents, and optimal surveillance methods for respiratory tract infections need to be determined. As emphasized by Drinka et al., we need better methods for the early detection of outbreaks, and in many situations a low threshold for beginning antiviral prophylaxis would be appropriate..$^{22}$ The use of rapid diagnostic testing would facilitate early identification and confirmation of influenza in a facility. Although the efficacy of isolation measures for interrupting the transmission of influenza in LTCFs has not been established, it would be prudent to confine ill residents to their rooms, emphasize good hand hygiene and appropriate use of gloves, limit or curtail group activities, restrict visitors from the community, and ensure that symptomatic staff are removed from patient care activities. ${ }^{13}$ Postexposure prophylaxis with antiviral agents is recommended to interrupt influenza transmission. Most experience has been with amantadine and rimantadine, but the study by Hirji et al. adds to the accumulating evidence suggesting that neuraminidase inhibitors are also safe and effective for LTCF residents. ${ }^{23}$ Preliminary results from the only study to compare a neuraminidase inhibitor with rimantadine during influenza outbreaks in LTCFs suggest that zanamivir or oseltamivir may have significant advantages over amantadine or rimantadine for the management of influenza A outbreaks. ${ }^{58}$ Neuraminidase inhibitors may also have an important role to play in LTCF outbreaks due to influenza B, but more clinical experience and evaluation of these drugs in this setting would be desirable. The potential merit of a strategy of seasonal prophylaxis with a neuraminidase inhibitor to prevent outbreaks versus postexposure prophylaxis once an outbreak has been identified should also be investigated. The articles by Drinka et al. and Hirji et al. in this issue of Infection Control and Hospital Epidemiology remind us that there is still ample opportunity for improving the management of influenza in LTCFs.

\section{REFERENCES}

1. Lui K-J, Kendal AP. Impact of influenza epidemics on mortality in the United States from October 1972 to May 1985. Am J Public Health 1987;77:712-716.

2. Simonsen L, Clarke MJ, Williamson GD, Stroup DF, Arden NH, Schonberger LB. The impact of influenza on mortality: introducing a severity index. Am J Public Health 1997;87:1944-1950.

3. Centers for Disease Control and Prevention. Prevention and control of influenza: recommendations of the Advisory Committee on Immunization Practices (ACIP). MMWR 2001;50(RR-4):1-46.

4. Goodman RA, Orenstein WA, Munro TF, Smith SC, Sikes K. Impact of influenza $\mathrm{A}$ in a nursing home. JAMA 1982;247:1451-1453.

5. Horman JT, Stetler HC, Israel E, Sorley D, Schipper MT, Joseph JM. An 
outbreak of influenza A in a nursing home. Am J Public Health 1986;76:501-504.

6. Meiklejohn $\mathrm{G}$, Hall $\mathrm{H}$. Unusual outbreak of influenza $\mathrm{A}$ in a Wyoming nursing home. I Am Geriatr Soc 1987;35:742-746.

7. Morens DM, Rash VM. Lessons from a nursing home outbreak of influenza A. Infect Control Hosp Epidemiol 1995;16:275-280.

8. Hall WN, Goodman RA, Noble GR, Kendal AP, Steece RS. An outbreak of influenza B in an elderly population. I Infect Dis 1981;144:297-302.

9. Simor AE, Sharpe S, Byrne S, Cruz T, McLaughlin B. Influenza B outbreak in a home for the aged, Ontario. Canada Diseases Weekly Report 1988; 14:181-184.

10. Stevenson CG, McArthur MA, Naus M, Abraham E, McGeer AJ. Prevention of influenza and pneumococcal pneumonia in long-term care facilities: how are we doing? Canadian Medical Association Journal 2001;164:1413-1419.

11. Gravenstein S, Miller BA, Drinka P. Prevention and control of influenza A outbreaks in long-term care facilities. Infect Control Hosp Epidemiol 1992;13:49-54

12. Gomolin IH, Leib HB, Arden NH, Sherman FT. Control of influenza outbreaks in the nursing home: guidelines for diagnosis and management. I Am Geriatr Soc 1995;43:71-74.

13. Bradley SF, the Long-Term-Care Committee of the Society for Healthcare Epidemiology of America. Prevention of influenza in longterm-care facilities. Infect Control Hosp Epidemiol 1999;20:629-637.

14. Patriarca PA, Weber JA, Parker RA, et al. Efficacy of influenza vaccine in nursing homes: reduction in illness and complications during an influenza A (H3N2) epidemic. JAMA 1985;253:1136-1139.

15. Patriarca PA, Weber JA, Parker RA, et al. Risk factors for outbreaks of influenza in nursing homes: a case-control study. $A m J$ Epidemiol 1986;124:114-119.

16. Meiklejohn G, Hoffman R, Graves P. Effectiveness of influenza vaccine when given during an outbreak of influenza $\mathrm{A} / \mathrm{H} 3 \mathrm{~N} 2$ in a nursing home. J Am Geriatr Soc 1989;37:407-410.

17. Foster DA, Talsma A, Furumoto-Dawson $A$, et al. Influenza vaccine effectiveness in preventing hospitalization for pneumonia in the elderly. Am J Epidemiol 1992;136:296-307.

18. McArthur MA, Simor AE, Campbell B, McGeer A. Influenza vaccination in long-term-care facilities: structuring programs for success. Infect Control Hosp Epidemiol 1999;20:499-503.

19. Arden NH. Control of influenza in the long-term-care facility: a review of established approaches and newer options. Infect Control Hosp Epidemiol 2000;21:59-64.

20. Potter J, Stott DJ, Roberts MA, et al. Influenza vaccination of health care workers in long-term-care hospitals reduces the mortality of elderly patients. J Infect Dis 1997;175:1-6.

21. Carman WF, Elder AG, Wallace $L A$, et al. Effects of influenza vaccination of health-care workers on mortality of elderly people in long-term care: a randomised controlled trial. Lancet 2000;355:93-97.

22. Drinka PJ, Krause P, Nest L, Gravenstein S, Goodman B, Shult P. Delays in the application of outbreak control prophylaxis for influenza $A$ in a nursing home. Infect Control Hosp Epidemiol 2002;23:600-603.

23. Hirji Z, O'Grady S, Bonham J, et al. Utility of zanamivir for chemoprophylaxis of concomitant influenza $A$ and $B$ in a complex continuing care population. Infect Control Hosp Epidemiol 2002;23:604-608.

24. McGeer A, Campbell B, Emori TG, et al. Definitions of infection for surveillance in long-term care facilities. Am I Infect Control 1991;19:1-7.

25. Gravenstein S, Miller BA, Ershler WB, et al. Low sensitivity of CDC case definition for H3N2 influenza in elderly nursing home subjects. Clinical Research 1990;38:547A.

26. Taylor JL, Dwyer DM, Coffman T, Groves C, Patel J, Israel E. Nursing home outbreak of influenza A (H3N2): evaluation of vaccine efficacy and influenza case definitions. Infect Control Hosp Epidemiol 1992;13:93-97.

27. Simor AE, Augustin A, Staynor $\mathrm{K}$, et al. Influenza $\mathrm{A}$ in elderly nursing home residents: evaluation of vaccine efficacy and case definitions of influenza-like illness. Presented at the 33rd Interscience Conference on Antimicrobial Agents and Chemotherapy; October 17-20, 1993; New Orleans, LA. Abstract 1383.

28. Orr PH, Peeling RW, Fast M, et al. Serologic study of responses to selected pathogens causing respiratory tract infection in the institutionalized elderly. Clin Infect Dis 1996;23:1240-1245.

29. Waner JL, Todd SJ, Shalaby H, Murphy P, Wall LV. Comparison of Directigen FLU-A with viral isolation and direct immunofluorescence for the rapid detection and identification of influenza A virus. $J$ Clin Microbiol 1991;29:479-482.

30. Leonardi GP, Leib H, Birkhead GS, Smith C, Costello P, Conron W Comparison of rapid detection methods for influenza $A$ virus and their value in health-care management of institutionalized geriatric patients. $J$ Clin Microbiol 1994;32:70-74.

31. Arden NH, Patriarca PA, Fasano MB, et al. The roles of vaccination and amantadine prophylaxis in controlling an outbreak of influenza A (H3N2) in a nursing home. Arch Intern Med 1988;148:865-868.

32. Staynor K, Foster G, McArthur M, McGeer A, Petric M, Simor AE.
Influenza A outbreak in a nursing home: the value of early diagnosis and the use of amantadine hydrochloride. Canadian Journal of Infection Control 1994;9:109-111.

33. Drinka PJ, Gravenstein S, Schilling M, Krause P, Miller BA, Shult P. Duration of antiviral prophylaxis during nursing home outbreaks of influenza A: a comparison of 2 protocols. Arch Intern Med 1998;158:2155-2159.

34. Degelau J, Somani S, Cooper SL, Irvine PW. Occurrence of adverse effects and high amantadine concentrations with influenza prophylaxis in the nursing home. J Am Geriatr Soc 1990;38:428-432.

35. Stange KC, Little DW, Blatnik B. Adverse reactions to amantadine prophylaxis of influenza in a retirement home. $J \mathrm{Am}$ Geriatr Soc 1991;39:700-705.

36. Keyser LA, Karl M, Nafziger AN, Bertino JS Jr. Comparison of central nervous system adverse effects of amantadine and rimantadine used as sequential prophylaxis of influenza $A$ in elderly nursing home patients. Arch Intern Med 2000;160:1485-1488.

37. Mast EE, Harmon MW, Gravenstein S, et al. Emergence and possible transmission of amantadine-resistant viruses during nursing home outbreaks of influenza A (H3N2). Am J Epidemiol 1991;134:988-997.

38. Degelau J, Somani SK, Cooper SL, Guay DRP, Crossley KB. Amantadineresistant influenza A in a nursing facility. Arch Intern Med 1992:152:390-392.

39. Houck P, Hemphill M, LaCroix S, Hirsh D, Cox N. Amantadine-resistant influenza $A$ in nursing homes: identification of a resistant virus prior to drug use. Arch Intern Med 1995;155:533-537.

40. Lee C, Loeb M, Phillips A, et al. Zanamivir use during transmission of amantadine-resistant influenza $\mathrm{A}$ in a nursing home. Infect Control Hosp Epidemiol 2000;21:700-704.

41. Hayden FG, Osterhaus ADME, Treanor JJ, et al. Efficacy and safety of the neuraminidase inhibitor zanamivir in the treatment of influenza virus infections. $N$ Engl J Med 1997;337:874-880.

42. The MIST (Management of Influenza in the Southern Hemisphere Trialists) Study Group. Randomised trial of efficacy and safety of inhaled zanamivir in treatment of influenza $A$ and $B$ virus infections. Lancet 1998;352:1877-1881.

43. Nicholson KG, Aoki FY, Osterhaus ADME, et al. Efficacy and safety of oseltamivir in treatment of acute influenza: a randomized controlled trial. Lancet 2000;355:1845-1850.

44. Treanor JJ, Hayden FG, Vrooman PS, et al. Efficacy and safety of the oral neuraminidase inhibitor oseltamivir in treating acute influenza: a randomized controlled trial. JAMA 2000;283:1016-1024.

45. Lalezari J, Campion K, Keene O, Silagyi C. Zanamivir for the treatment of influenza A and B infection in high-risk patients: a pooled analysis of randomized controlled trials. Arch Intern Med 2001;161:212-217.

46. Monto AS, Robinson DP, Herlocher ML, Hinson JM Jr, Elliott MJ, Crisp A. Zanamivir in the prevention of influenza among healthy adults: a randomized controlled trial. JAMA 1999;282:31-35.

47. Hayden FG, Atmar RL, Schilling M, et al. Use of the selective oral neuraminidase inhibitor oseltamivir to prevent influenza. $N$ Engl $\mathrm{J} \mathrm{Med}$ 1999:341:1336-1343

48. Hayden FG, Gubavera LV, Monto AS, et al. Inhaled zanamivir for the prevention of influenza in families. N Engl J Med 2000;343:1282-1289.

49. Welliver R, Monto AS, Carewicz O, et al. Effectiveness of oseltamivir in preventing influenza in household contacts: a randomized controlled trial. JAMA 2001;285:748-754.

50. Calfee DP, Hayden FG. New approaches to influenza chemotherapy: neuraminidase inhibitors. Drugs 1998;56:537-553.

51. Barnett JM, Cadman A, Gor D, et al. Zanamivir susceptibility monitoring and characterization of influenza virus clinical isolates obtained during phase II clinical efficacy studies. Antimicrob Agents Chemother 2000;44:78-87.

52. Gubavera LV, Kaiser L, Matrosovich MN, Soo-Hoo Y, Hayden FG. Selection of influenza virus mutants in experimentally infected volunteers treated with oseltamivir. / Infect Dis 2001;183:523-531.

53. Lee C, Loeb M, Phillips A, et al. Zanamivir use during transmission of amantadine-resistant influenza $\mathrm{A}$ in a nursing home. Infect Control Hosp Epidemiol 2000;21:700-704.

54. McGeer AJ, Lee W, McArthur M, et al. Use of zanamivir to control an outbreak of influenza A in a nursing home. Clin Infect Dis 2000;31:318.

55. Bowles SK, Lee W, Simor AE, et al. Use of oseltamivir during influenza outbreaks in Ontario nursing homes, 1999-2000. I Am Geriatr Soc 2002;50:608-616.

56. Peters PH Jr, Gravenstein S, Norwood P, et al. Long-term use of oseltamivir for the prophylaxis of influenza in a vaccinated frail older population. J Am Geriatr Soc 2001;49:1025-1031.

57. Schilling M, Povinelli L, Krause P, et al. Efficacy of zanamivir for chemoprophylaxis of nursing home influenza outbreaks. Vaccine 1998;16:1771-1774.

58. Gravenstein S, Drinka P, Osterweil D, et al. A multicenter prospective double-blind randomized controlled trial comparing the relative safety and efficacy of zanamivir to rimantadine for nursing home influenza outbreak control. Presented at the 40th Interscience Conference on Antimicrobial Agents and Chemotherapy; September 17-20, 2000; Toronto, Ontario, Canada. Abstract 1155 